\title{
Enabling Ultra-small Sensor Spacecraft for the Space Environment using Small-Scale Electrodynamic Tethers
}

\author{
Iverson C. Bell, III $^{1}$ and Brian E. Gilchrist ${ }^{2}$ \\ The University of Michigan, Ann Arbor, Michigan 48109, USA \\ Jesse K. McTernan ${ }^{3}$ and Sven G. Bilén ${ }^{4}$ \\ The Pennsylvania State University, University Park, Pennsylvania 16802, USA \\ Robert P. Hoyt ${ }^{5}$ and Nestor R. Voronka ${ }^{6}$ \\ Tethers Unlimited, Inc. Bothell, Washington 98011, USA \\ and \\ Mason A. Peck ${ }^{7}$ \\ Cornell University, Ithaca, New York 14853, USA
}

\begin{abstract}
In this paper we investigate an approach that appears to scale to the small size needed for femtosatellite (commonly called "ChipSats") drag make-up and even orbit raising with the added benefit of being propellantless. The approach uses a short, semi-rigid electrodynamic tether (EDT) for propulsion, which keeps the overall ChipSat mass low and provides enough thrust to overcome drag in LEO. We report on our trade studies to assess the feasibility of using the EDT for ChipSat propulsion. We have analyzed the EDT anode's ability to draw current from the ionosphere and thereby generate thrust. We then traded this performance against the tether mass and material, electron emitter and collector types, and power needed to determine the EDT's capability of overcoming atmospheric drag forces. The results reveal that an insulated tether only a few meters long and tens of microns in diameter could provide milligram to 100 gram-level ChipSats with complete drag cancellation and even the ability to change orbit. The EDT system described here might be considered inefficient in terms of the power required for thrust. However, the received solar power is sufficient and the EDT is propellantless, so we believe the EDT still provides a viable approach for propulsion. We also explore the assumption that the gravity gradient force aligns the tether along the local vertical and find that this assumption needs further investigation. A more complete systems design and analysis is continuing.
\end{abstract}

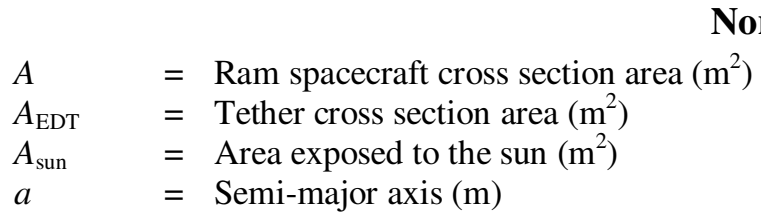

\footnotetext{
${ }^{1}$ Ph.D. Candidate, Electrical Engineering, 1301 Beal Ave., Student Member.

${ }^{2}$ Professor, Electrical Engineering and Computer Science, 1301 Beal Ave., Associate Fellow.

${ }^{3}$ Graduate Student Research Assistant, 229 Hammond Building, Student Member.

${ }^{4}$ Assoc. Professor, Engineering Design, Electrical Engineering, and Aerospace Engineering, 213 Hammond Bldg., Associate Fellow.

${ }^{5}$ CEO and Chief Scientist, 11711 North Creek Parkway South, Suite D-113, Senior Member.

${ }^{6}$ VP and Chief Technologist, 11711 North Creek Parkway South, Suite D-113, Senior Member.

${ }^{7}$ Associate Professor, Mechanical and Aerospace Engineering, 212 Upson Hall, Member.
} 


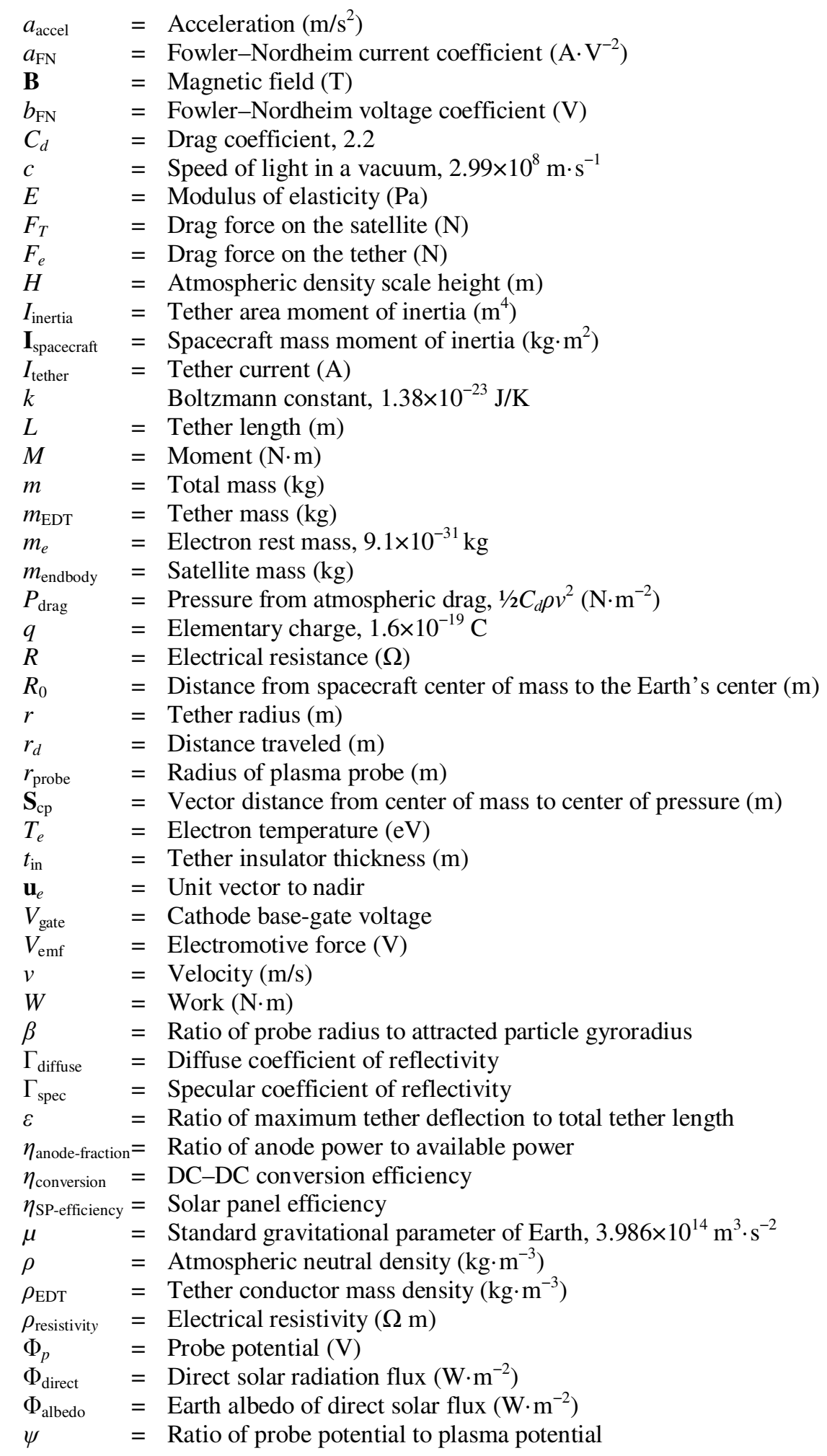

\section{Introduction}

7 he growing success of and interest in nanospacecraft (1-10 kg) over the past decade has generated interest in exploring the potential for even smaller spacecraft, both as stand-alone satellites or as a distributed $s w a r m .{ }^{1-3}$ 
Because of advances in integrated circuit and microelectromechanical systems (MEMS) technology, the feasibility of miniaturized spacecraft at the levels of fully monolithic semiconductor integrated circuits (10-100 mg) or hybrid integrated circuits $(10-100 \mathrm{~g})$ is being seriously investigated. ${ }^{4-6}$ ChipSats belong to the picosatellite $(100 \mathrm{~g}-1 \mathrm{~kg})$ and femtosatellite $(<100 \mathrm{~g})$ mass categories. Effectively, this architecture can be thought of as a small "satellite-ona-chip" or "ChipSat".

Because of their low masses and small sizes, they are orders of magnitude less costly to manufacture, test, and boost into orbit. Large groups of reconfigurable satellites also present unique mission capabilities, such as simultaneous, distributed data collection; ad hoc in-space data relay networking; and enhanced communication via configuration as an antenna array.

Flat ChipSat wafers, however, have an inherently high area-to-mass ratio. Although this feature can be exploited for new behaviors, it can result in an undesirably short orbital lifetime in low Earth orbit (LEO) due to atmospheric drag, ranging from a few days to a few hours depending on altitude and solar conditions. While a satellite using chemical propulsion can overcome the continuous force of atmospheric drag, the volume of propellant required will increase with the satellite's intended lifetime. Thus, the use of a traditional thruster with propellant and need for directed flow to compensate for drag and possibly for maneuverability would increase the size, mass, and complexity of ChipSats. The need for ChipSat maneuverability is particularly important considering the technology's capability for swarm missions that might require a high degree of orbital maintenance. The small size of the satellites also presents a challenge for energy storage, communication, and tracking.

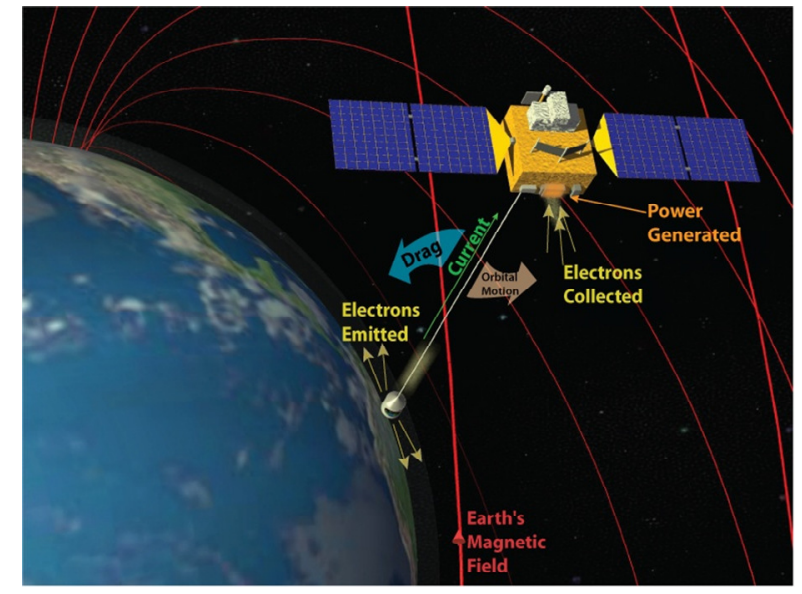

Figure 1. Illustration of electrodynamic tether operating, attached to a larger sized spacecraft in orbit.

In this paper, we investigate an approach that appears to scale to the small size needed and is also propellantless. The approach uses a short, semi-rigid electrodynamic tether (EDT) for propulsion, illustrated in Fig. 1, which keeps the overall ChipSat mass low and provides enough thrust to overcome drag in LEO. An EDT exploits the Lorentz force to generate thrust for boost, deboost, and inclination change, using current in a conducting tether to produce a force in the presence of the Earth's magnetic field. The Lorentz force is expressed as ${ }^{8}$

$$
\mathbf{F}_{\text {Lorentz }}=\int_{0}^{L}\left(I_{\text {tether }} d \mathbf{L}\right) \times \mathbf{B}
$$

and the magnitude of the force is

$$
F_{\text {Lorentz }}=I_{\text {tether }} L B
$$

for a straight, insulated tether oriented perpendicular to the magnetic field.

The tether circuit is closed by collecting charge from the Earth's ionosphere at one end and emitting charge of the same sign or collecting charge of the opposite sign at the other end, with final closure occurring in the surrounding plasma. This process is illustrated in Fig. 1.

We report on our trade studies to assess the feasibility of using the EDT for ChipSat propulsion. We have analyzed

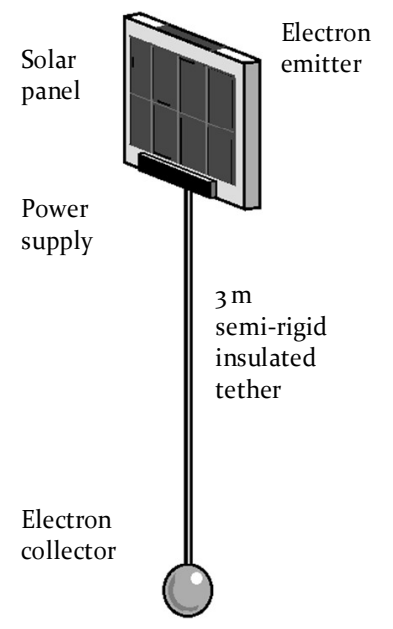

Figure 2a. Early EDT concept. ${ }^{7}$

\section{ChipSat}

Figure 2b. Advanced ChipSat EDT concept. Each end-body has a solar panel, power supply, and an electron emitter and is capable of collecting electrons on the surface. ${ }^{9}$ 
the EDT anode's ability to draw current from the ionosphere and through the tether, thereby generating thrust, and have traded this performance against the tether mass and material, tether rigidity, and power needed to determine the EDT's capability of overcoming atmospheric drag forces. The study led to the development of a system concept and mission scenario using the simulation tool TeMPEST to estimate tether voltages and currents based on tether configuration and ambient models. TeMPEST incorporates current geomagnetic field models, ionospheric and atmospheric conditions, plasma contactor modeling, and precise orbital calculations.

The system concept developed in the previous trade study, ${ }^{7}$ shown in Fig. $2 \mathrm{a}$, is capable of drag make-up and boost. The ChipSat is also oriented so the maximum cross-sectional area is perpendicular to velocity. Figure $2 \mathrm{~b}$ shows the more advanced system concept being investigated. ${ }^{9}$ This system has the capability to boost, deboost, and collect solar energy with the upper and lower end-bodies. The satellite is oriented so the minimum cross-sectional area is perpendicular to velocity. We designed the satellite-tether symmetry to position the center of pressure, the center of mass, and the center of gravity roughly at the same location midway along the tether. This should help reduce torque introduced by atmospheric drag.

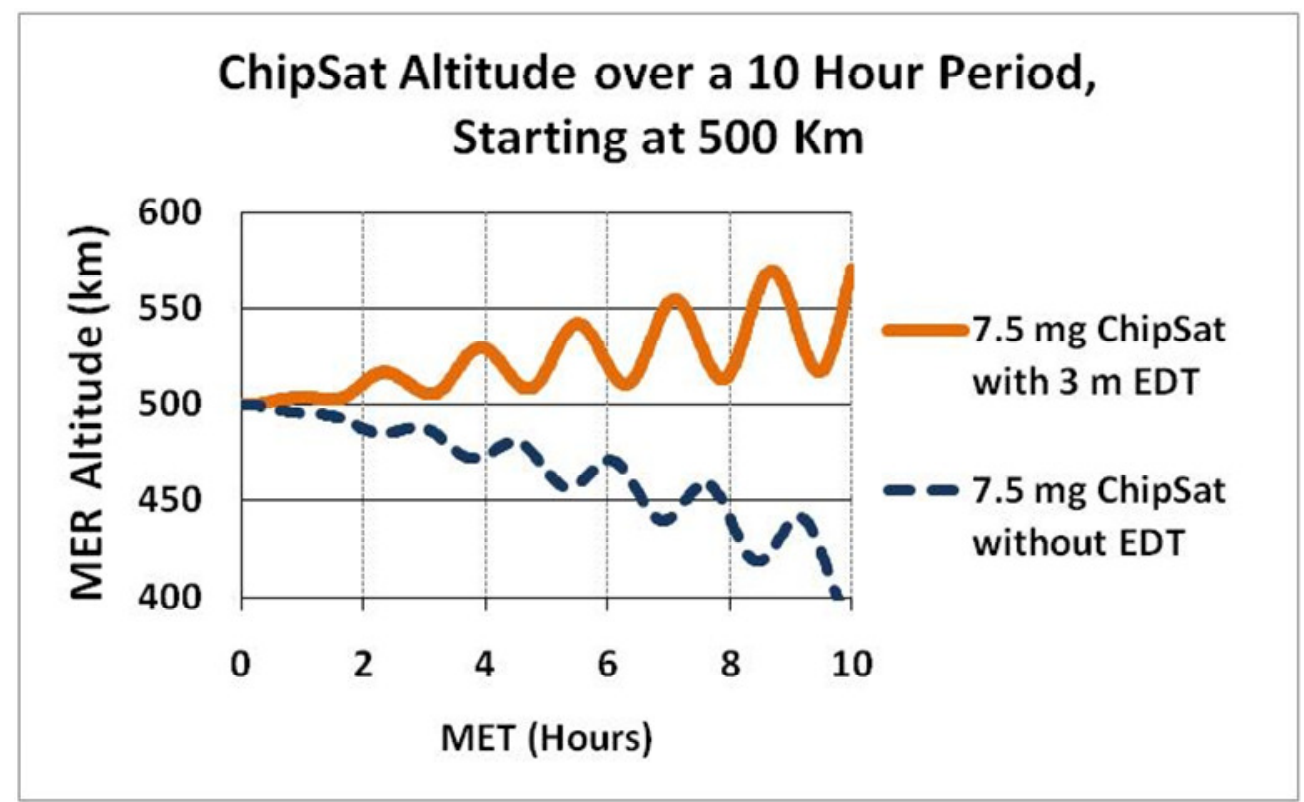

Figure 3. TeMPEST simulation results of ChipSat orbit with and without the electrodynamic tether.

The results from the previous trade study, ${ }^{7}$ shown in Fig. 3, reveal that an insulated tether only a few meters long and tens of microns in diameter can provide milligram- to 100-gram-level ChipSats with complete drag cancellation and even the ability to change orbit. Additional details on the trade study's assumptions and calculations may be found in Ref. 7.

\section{Trade Study}

\section{A. Ionosphere/Atmosphere Environment}

The conditions of the atmosphere and ionosphere heavily influence EDT performance. The current in the tether, $I_{\text {tether }}$, scales with electron density. The peak electron density, and thus EDT thrust capability, occurs in the F2 region of the ionosphere between 300 and $500 \mathrm{~km}$. We assume a 500-km circular orbit. We also assume a low inclination orbit to ensure that the thrust in Eq. (1) is primarily in-plane for a tether oriented along the local vertical.

We conducted the trade study during high solar activity, when the neutral-to-electron-density ratio is an order of magnitude higher than at low solar activity. The force of atmospheric drag counters orbital velocity and reduces the altitude of an orbiting body over time. The expression for atmospheric drag force is given by

$$
\mathbf{F}_{\mathrm{drag}}=-\frac{1}{2} \rho C_{d} A v^{2} \hat{\mathbf{v}}
$$

and torque due to drag is given by ${ }^{10}$ 


$$
\boldsymbol{\tau}_{\mathrm{drag}}=\mathbf{F}_{\mathrm{drag}} \times \mathbf{S}_{\mathrm{cp}}
$$

The drag force scales with the neutral density and the thrust scales with electron density, so the elevated neutral-to-electron-density ratio during high solar activity actually represents the worst case scenario for thrusting at $500 \mathrm{~km}$.

We determined the electron density by averaging the densities calculated at $500 \mathrm{~km}$ altitude at the equator during the solar high of solar cycle 23 (July 2000 ${ }^{11}$ ) using the International Reference Ionosphere-2007 (IRI2007) model. The neutral density was similarly taken from the Mass-Spectrometer-Incoherent-Scatter (MSISE-90) model. Atmosphere and ionosphere assumptions are summarized in Table 1.
Table 1. Ionospheric conditions.

\begin{tabular}{|c|c|}
\hline Parameter & Value \\
\hline Altitude & $500 \mathrm{~km}($ circular $)$ \\
\hline $\begin{array}{c}\text { Spacecraft Velocity (relative to } \\
\text { co-rotating atmosphere) }\end{array}$ & $7.5 \mathrm{~km} \cdot \mathrm{s}^{-1}$ \\
\hline F10.7 (solar activity) & $169($ Solar High) \\
\hline Electron Temperature & $0.15 \mathrm{eV}$ \\
\hline Magnetic Field & $0.3 \mathrm{gauss}$ \\
\hline Gyroradius & $3 \mathrm{~cm}$ \\
\hline Neutral Density & $1 \times 10^{-15} \mathrm{~g} \cdot \mathrm{cm}^{-3}$ \\
\hline Electron Density & $5 \times 10^{5} \mathrm{electrons} \cdot \mathrm{cm}^{-3}$ \\
\hline Debye Length & $4 \mathrm{~mm}$ \\
\hline Electron-to-neutral Density & $1 \times 10^{22}$ electrons $\cdot \mathrm{g}^{-1}$ \\
\hline
\end{tabular}

\section{B. Orbital Lifetime}

The atmospheric drag force is the dominant non-gravitational force in LEO. The change in semi-major axis due to atmospheric drag is given by ${ }^{10}$

$$
\Delta a_{\text {revolution }}=2 \pi\left(\frac{C_{d} A}{m}\right) \rho a^{2}
$$

which scales with the satellite's area-to-mass ratio. The orbital lifetime

$$
\text { Lifetime } \approx \frac{H}{\Delta a_{\text {revolution }}}
$$

is roughly proportional to the inverse of this ratio, or the ballistic coefficient. Figure 4 illustrates that smaller femtosatellites have lower ballistic coefficients. The estimated lifetimes of the satellites in Fig. 2 in LEO range from years for the Surrey SpaceChip ${ }^{4}$ to days for the Cornell MCM Sat. ${ }^{5}$ In order to make formation flying more feasible and to extend the orbital lifetime of the spacecraft, there is a strong benefit for a propulsion system that scales to the size of the satellites without significantly increasing the satellites' onboard mass, power, or complexity.

\section{Trade Study Satellites}

The satellites in the trade study roughly span the mass and ballistic coefficient range represented by actual pico- and femtosatellites, as shown in Fig. 4. The mass and size of the largest satellite in the trade study (100 g) was motivated by the DARPA/Aerospace Corporation satellites PicoSat 1 and 2. PicoSats 1 and 2 were identical 250 -g satellites connected by a 30 meter tether. They were launched in January

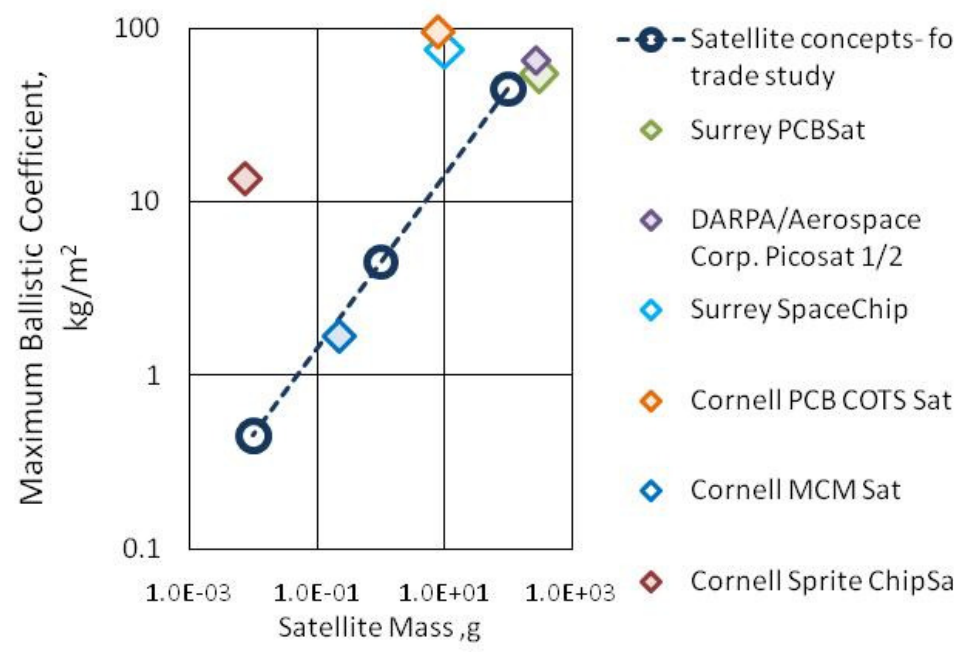

Figure 4. Ballistic coefficient for a range of ultra-small satellites assuming the smallest area cross-section. ${ }^{4,5,6}$ $2000{ }^{6}$

The mission demonstrated RF-MEMS in space. Each PicoSat was equipped with a radio and could communicate to the other satellite and to the ground. The Picosats could also be successfully tracked because the 
Table 2. Parameters for satellites used in the trade study.

\begin{tabular}{|c|c|c|c|c|c|}
\hline $\begin{array}{c}\text { Satellite concept for } \\
\text { trade study }\end{array}$ & Dimensions & Mass & $\begin{array}{c}\text { Cross section } \\
\text { area }\end{array}$ & $\begin{array}{c}\text { Drag force, 500- } \\
\text { km altitude }\end{array}$ & $\begin{array}{c}\text { Orbital lifetime estimate, } \\
\text { 500-km initial altitude }\end{array}$ \\
\hline Large femtosatellite & $2 \mathrm{~cm} \times 5 \mathrm{~cm} \times 5 \mathrm{~cm}$ & $100 \mathrm{~g}$ & $10 \mathrm{~cm}^{2}$ & $60 \mathrm{nN}$ & few years \\
\hline Medium femtosatellite & $1 \mathrm{~cm} \times 1 \mathrm{~cm} \times 1 \mathrm{~cm}$ & $1 \mathrm{~g}$ & $1 \mathrm{~cm}^{2}$ & $6 \mathrm{nN}$ & few months \\
\hline Small femtosatellite & $1 \mathrm{~mm} \times 1 \mathrm{~cm} \times 1 \mathrm{~cm}$ & $10 \mathrm{mg}$ & $0.1 \mathrm{~cm}^{2}$ & $0.6 \mathrm{nN}$ & few days \\
\hline
\end{tabular}

tether connecting the PicoSats had a thin gold wire strand that increased the radar cross section of the structure. The ground antenna tracking the satellites was a 150 -ft-diameter $(45.7-\mathrm{m})$ parabolic antenna. ${ }^{12}$

The smallest satellite considered in the study, the $10-\mathrm{mg}$ satellite, was motivated by the Sprite ChipSat. ${ }^{5}$ The Sprite ChipSat is currently undergoing testing on the Materials International Space Station Pallet on the International Space Station. The 1-g femtosatellite bridges the mass and ballistic coefficient gap between the higher and lower mass satellites. Information on the satellites used in the trade study is provided in Table 2.

\section{Tether Material Composition}

The tether for a miniaturized EDT will have different requirements than tethers used for more massive satellites. In an EDT system for larger spacecraft, one satellite, or end-body, deploys a second end-body and the two are connected by a tether cable that can range from a few hundred meters to tens of kilometers in length. We expect a tether for femtosatellites to be much shorter. The end-bodies for traditional EDT systems are also orders of magnitude larger and more massive than considered here. For example, in the Tethered Satellite System (TSS) missions, the Space Shuttle, which has a mass of about 100,000 kg, deployed a $521 \mathrm{~kg}, 1.6-\mathrm{m}$ diameter sphere as the second end-body. ${ }^{13}$ In this case, the gravity-gradient force generated tension along the tether, pulling the long cable along the local vertical. The approximate gravity gradient force is given by ${ }^{10}$

and the corresponding torque is given by ${ }^{10}$

$$
F_{\text {gravity-gradient }} \approx \frac{3 m \mu L}{R_{0}^{3}}
$$

$$
\boldsymbol{\tau}_{\text {gravity-gradient }}=\frac{3 \mu}{R_{0}^{3}} \mathbf{u}_{e} \times\left(\mathbf{I}_{\text {spacecraft }} \bullet \mathbf{u}_{e}\right)
$$

The gravity gradient torque can orient long objects along the local vertical and the force given by Eq. (7) can pull long, flexible structures roughly straight. Vertical orientation is important because a tether that is straight and aligned along the local vertical in a low inclination orbit in LEO can generate peak in-plane thrust.

In our design, we assume the end-bodies are identical femto- or picosatellites of equal size and mass since current collection and emission must occur at each end of the tether. The vertical symmetry also helps improve the ability to have the center of mass, center of gravity, and the center of pressure located roughly at the midway point along the tether. This should help reduce any torques induced by atmospheric drag, like that given in Eq. (4). The gravity-gradient force given by Eq. (7), which scales with mass and length, will be small for femtosatellites. As a result, a more rigid material is required to ensure that the tether achieves near straight orientation regardless of forces along its length or on the end-bodies, e.g., drag or solar pressure. On a long time scale, if the tether acts like a rigid beam, the gravity-gradient torque in Eq. (8) should align it in the local vertical assuming symmetry in the system. We consider this assumption further in section $\mathrm{K}$.

We have designed the EDT to have a semirigid, conducting metal core and a thin layer of insulation. We chose Kapton $^{\mathrm{TM}}$ to be the insulation material due to its common use as spacecraft insulation and its high breakdown potential. The tether insulation is $2 \mu \mathrm{m}$ thick. In

Table 3. Properties of tether materials used for the trade study.

\begin{tabular}{|c|l|}
\hline Material Property & \multicolumn{1}{|c|}{ Value } \\
\hline Monel K-500 $^{14}$ & \\
\hline Electrical resistivity $\left(21^{\circ} \mathrm{C}\right)$ & $6.15 \times 10^{-7} \Omega \cdot \mathrm{m}$ \\
\hline Mass density & $8.47 \mathrm{~g} \cdot \mathrm{cm}^{-3}$ \\
\hline Elastic Modulus, Tension & $179 \mathrm{GPa}$ \\
\hline Kapton film $^{15}$ & \\
\hline Dielectric Strength & $291 \mathrm{~V} \cdot \mu \mathrm{m}^{-1}$ \\
\hline Mass density & $1.54 \mathrm{~g} \cdot \mathrm{cm}^{-3}$ \\
\hline
\end{tabular}
the case that the anode bias voltage approaches the breakdown potential for $2-\mu \mathrm{m}$ insulation, we instead use $4 \mu \mathrm{m}$ of Kapton. 
We chose Monel-K500 to be the EDT core because of its high Young's modulus and yield strength. Although Monel $^{\mathrm{TM}}$ is not a good conductor, the tether lengths and currents are small enough that the resistance, given by

$$
R=\rho_{\text {resistivity }}\left(\frac{L}{A}\right)
$$

is not high. Furthermore, the power dissipated in the tether is not a dominant factor because this loss term scales with resistance and the square of current, both of which are small values. Some properties of Kapton film and Monel-K500 are included in Table 3.

\section{E. Tether Rigidity and Radius}

The force of atmospheric drag can bend or bow a tether because the magnitude of this force can vary between the tether and the end-bodies. Sufficient bowing reduces the vertical length of the tether, which in turn can reduce thrust from that in Eq. (2). To investigate tether bowing due to drag, we use D'Alembert's principle to transform the accelerating body into a static system by adding inertial forces and torques. This technique allows us to solve for EDT deflection as we would solve for deflection along a simply supported beam. ${ }^{16}$ The details of the derivation may be found in Ref. 9. The variation in drag force along the structure causes a maximum deflection at the center calculated by

$$
y_{\text {max }}=-\frac{10 L^{3} P_{\text {drag }}}{384 E I_{\text {inertia }}}\left(\frac{A_{\mathrm{EDT}} m_{\text {endbody }}-A m_{\mathrm{EDT}}}{2 m_{\text {endbody }}+m_{\mathrm{EDT}}}\right) .
$$

The deflection $y_{\max }$ in Eq. (10) gives the bowing distance, which is the distance the center of the tether moves relative to the end-bodies. The zeros of Eq. (10), or tether radii that cause $y_{\max }=0$, cannot be used because the radii are either extremely small (sub-micron) or large (millimeter). Alternatively, we can solve for a radius in Eq. (10) that limits tether bowing to a small distance. We solve Eq. (10) by limiting the maximum deflection to the total length multiplied by a small number $\varepsilon$, i.e., $y_{\max }=\varepsilon L$. For example, if $\varepsilon=0.01$ for a $1-\mathrm{m}$ tether, the tether center bends outward $1 \mathrm{~cm}$ from both ends. Equation (10) can be rearranged to give the atmospheric drag pressure

$$
P_{\text {drag }}=\varepsilon 38.4 \frac{E I_{\text {inertia }}}{L^{2}}\left(\frac{2 m_{\text {endbody }}+m_{\mathrm{EDT}}}{A_{\mathrm{EDT}} m_{\text {endbody }}-A m_{\mathrm{EDT}}}\right) .
$$

We rewrite Eq. (11) as

$$
\frac{1}{2} C_{d} \rho v^{2}=\varepsilon 38.4 \frac{E\left(\frac{\pi}{4}\left(r-t_{\text {in }}\right)^{4}\right)}{L^{2}}\left(\frac{2 m_{\text {endbody }}+\left(\rho_{\mathrm{EDT}} L \pi\left(r-t_{\text {in }}\right)^{2}\right)}{2 r L m_{\text {endbody }}-A\left(\rho_{\mathrm{EDT}} L \pi\left(r-t_{\text {in }}\right)^{2}\right)}\right)
$$

in order to solve for the radius of the tether. One should note that the radius depends on ambient conditions.

Figure 5 shows the radii for tether stiffness for a range of tether lengths. It is important to note that, in the remainder of the trade study, we calculate parameters for each satellite such as drag, current, and power using the EDT radii in Fig. 5 that make the tether stiff at a given length. For example, a 10-m-long EDT designed for a 100-g satellite $(\varepsilon=0.01, r=106 \mu \mathrm{m})$ has a different radius than a 1-m-long EDT designed for the same satellite $(\varepsilon=0.01$, $r=24 \mu \mathrm{m})$. However, both tethers bow outwards a maximum of $1 \%$ the total length due to atmospheric drag. The insulator is very thin and flexible relative to the metal core, so we assume that the Monel core provides the rigidity.

We assume that lateral deflection where $\varepsilon=0.01$ is sufficient to treat the tether and satellites as a single rigid structure. To illustrate the impact of the relationship between radius and required current, we write Eq. (2) as

$$
I_{\text {tether }}=\frac{F_{\text {drag }}}{L B}=\frac{\frac{1}{2} c \rho v^{2}(2 A+2 L r)}{L B} .
$$


The current required for drag makeup scales with tether length and radius. Stiffer tethers have larger radii and, consequently, a higher drag force and required current. Thus, if one designs an EDT with a large radius to further increase stiffness, the system requires more current to overcome the elevated drag force.

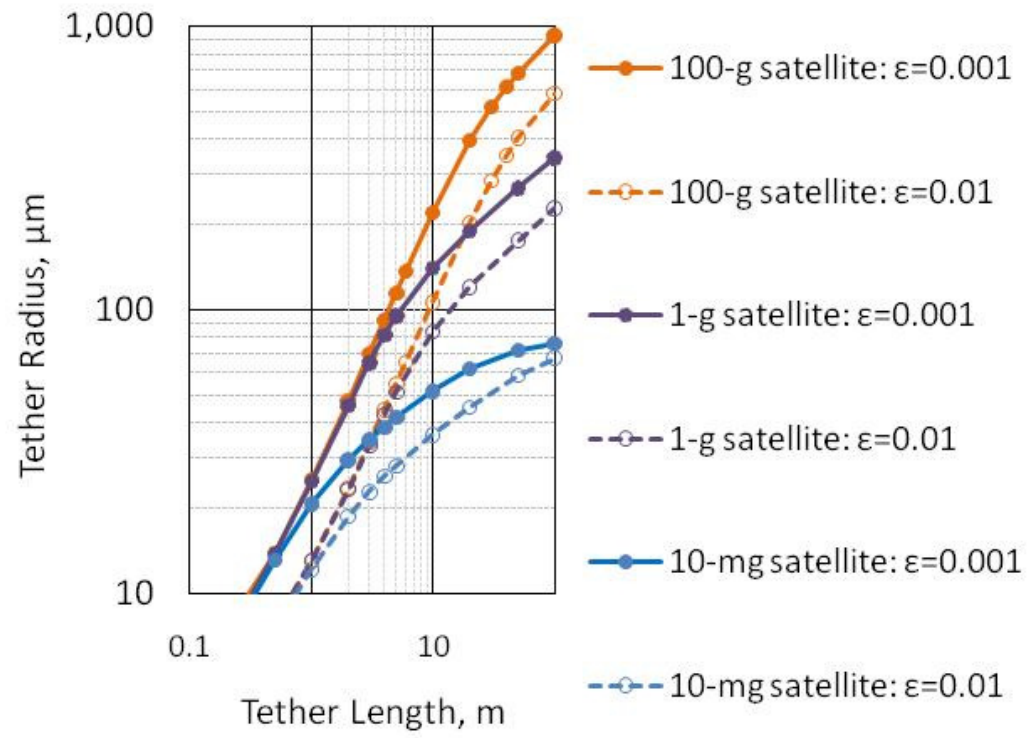

Figure 5. Minimum radius for tether rigidity.

\section{F. Tether Mass}

The tether mass can be calculated for a given length by

$$
m_{\mathrm{EDT}}=\rho_{\mathrm{EDT}} L \pi r^{2}
$$

using the tether radii found in Fig. 5 and the material densities found in Table 3. The mass reported in Fig. 6 shows the mass of the tether and the mass of the satellite. The lower end-body is a femtosatellite, so the total mass of the system is the sum of the tether mass and twice the satellite mass.

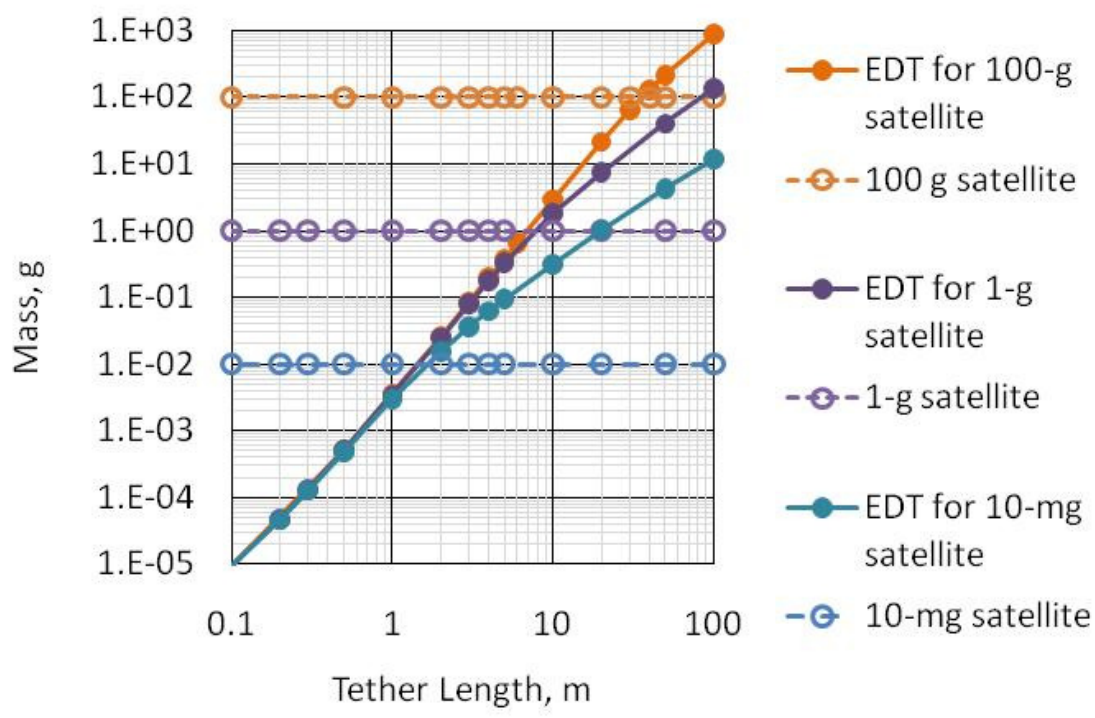

Figure 6. Tether and satellite mass. 


\section{G. Tether Current}

\section{Anode current}

EDT thrust is proportional to current. Due to the higher mobility of electrons, more electron current may be collected per unit area than ion current. Electrons can be collected on the outer surfaces of the satellites. We assume that the femtosatellites can be coated with a transparent conductor, e.g., indium tin oxide, to facilitate the needed low current collection. For the 1-g and 10mg femtosatellite, we can roughly estimate the current by approximating the satellite to be a sphere with a diameter equal to each satellite's largest dimension or diagonal. The diagonals of the 10-mg and 1-g satellites are about 3 Debye lengths For an anode at a high bias with respect to plasma potential $\left(\sim \Phi_{p}>\right.$ $100 T_{e}$ or $\left.15 \mathrm{~V}\right)$, we expect the sheath to extend outwards several Debye lengths into the plasma and shield out the precise probe geometry.

We conservatively estimate current collected by the 100-g satellite by assuming all four 2-cm-high edges of the satellite collect current like a single 2$\mathrm{cm}$-diameter sphere. The six faces should collect current like flat plates, but the current collection on these surfaces is small. We also ignore collection along the eight $5-\mathrm{cm}$ satellite edges. It should be noted that this estimate may be too conservative and should be analyzed further.

The Rubinstein-Laframboise (R-L) canonical upper bound current can estimate current collected by a biased spherical probe in a weakly magnetized plasma. The R-L current is given by ${ }^{17}$

$$
I_{\mathrm{R}-\mathrm{L}}=I_{\text {tether }}=I_{\text {thermal }}\left(\frac{1}{2}+\frac{2 \sqrt{\psi}}{\beta \sqrt{\pi}}+\frac{2}{\beta^{2} \pi}\right), \psi \rightarrow \infty,
$$

where the thermal current is

$$
I_{\text {thermal }}=4 \pi r_{\text {probe }}^{2} n_{e} q \sqrt{\frac{k T_{e}}{2 \pi m_{e}}}
$$

And the normalized bias is

$$
\psi=\frac{\Phi_{p}-V_{\text {plasma }}}{T_{e}} .
$$

Equation (15) is an approximation of the R-L current that assumes $\Phi_{p}>>T_{e}$. Figure 7 shows the R-L currentvoltage $(I-V)$ curve relative to the orbital motion limited ${ }^{18}$ (OML) and Parker-Murphy ${ }^{19}$ (P-M) $I-V$ curves. The Parker-Murphy theory is appropriate when the probe-radius-to-gyroradius ratio, or $\beta$, far exceeds the $\beta$ for small satellites in LEO. Thus, we expect to collect much more current at a given voltage than this theory and less than the maximum allowable current estimated by OML theory. The R-L $I-V$ curve lies between the OML and P-M curves, so it is our best estimate.

\section{Cathode current}

Field emitter array (FEA) technology can be used to emit electrons at the opposite end of the tether. The Fowler-Nordheim emission law is ${ }^{20}$

$$
I_{\text {cathode }}=a_{F N} V_{\text {gate }}^{2} \exp \left(-b_{F N} / V_{\text {gate }}\right)
$$


The Spindt cathode consists of an array of sharp-tipped, sub- $\mu$ m-radius cones that emit electrons when the nearby gate is biased to $V_{\text {gate }}$. Carbon nanotube emitter arrays may be a feasible alternative for future femtosatellites. A minimum current is noticeable at specific EDT lengths in Fig. 5: the current minimum is 1 meter for the 10-mg satellite, 3 meters for the 1 -g satellite, and 10 meters for the 100-g satellite. We calculate the maximum available current, shown in Fig. 8, from available power, which we discuss in Section $\mathrm{H}$.

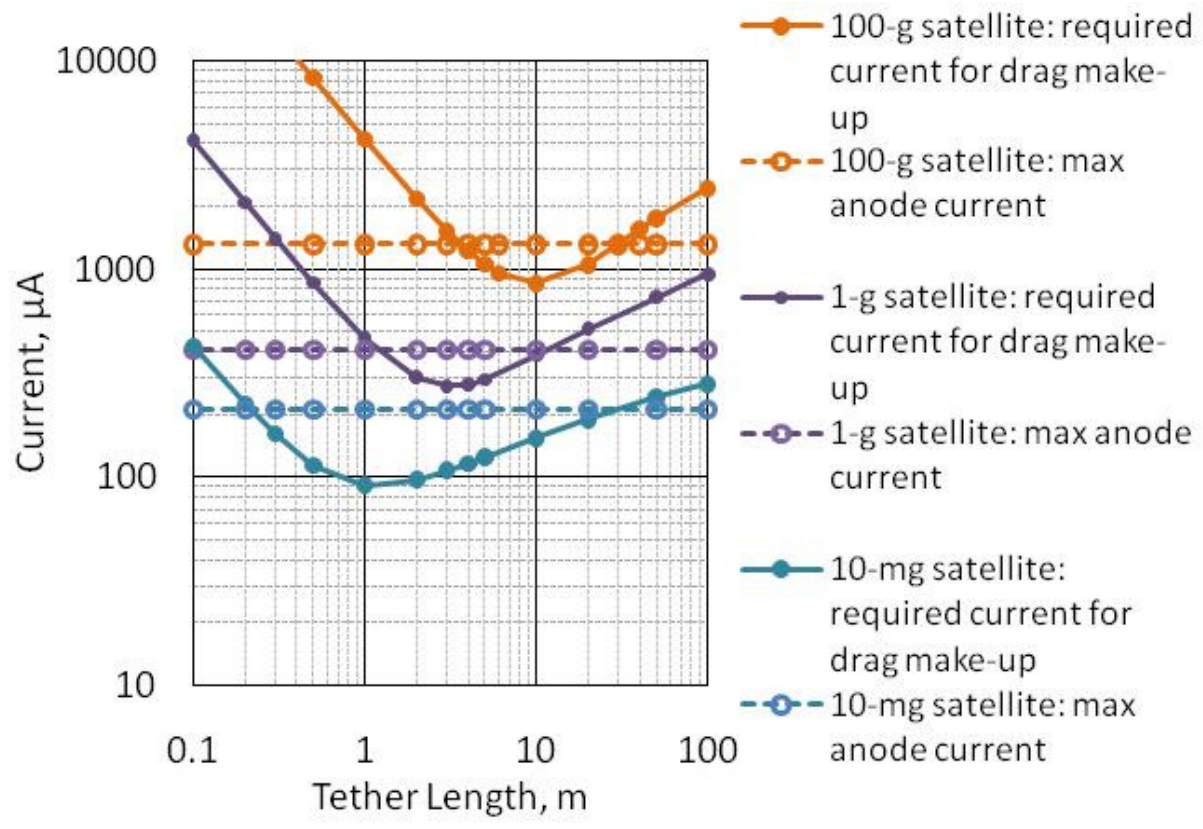

Figure 8. Required current for rigid tether $(\varepsilon=0.01)$. 


\section{H. Power}

In order to determine if EDT propulsion is feasible for femtosatellites, the electrical power used by the EDT system to overcome the drag force must be less than the generated power.

\section{Generated power}

We estimate the amount of electrical power generated by the satellite with the expression

$$
P_{\text {generated }}=\frac{1}{2} \eta_{\text {SP-efficiency }}\left(A_{\text {sun }} \phi_{\text {direct }}+A_{\text {sun }} \phi_{\text {albedo }}\right) \text {. }
$$

We assume that the femtosat has solar panels on all six sides of the upper and lower end-bodies, three of which are exposed to the sun at any given time. The solar panel has an efficiency $\eta_{\text {SP-efficiency, which we assume to be } 10 \% \text { as }}$ reported in Ref. 21. Of the total energy collected from direct solar radiation and Earth albedo, we assume that $25 \%$ is lost in the step-up voltage DC-DC converter and other loads. We neglect the small energy contribution from Earth infrared radiation. The expression estimating generated power also assumes that the satellite is in the sun roughly half of the time, i.e., the $1 / 2$ coefficient. The actual amount of time in the sun depends on the orbit and the altitude.

2. Dissipated power

The power required for EDT thrust is

$$
P_{\text {total }}=P_{\text {tether }}+P_{\text {anode }}+P_{\text {cathode }} \text {. }
$$

The power required to collect electrons is a fraction of the total available power, given by

and it can be calculated by

$$
P_{\text {anode }}=\eta_{\text {anode-fraction }} P_{\text {generated }},
$$

$$
P_{\text {anode }}=I_{\mathrm{R}-\mathrm{L}} \Phi_{p} .
$$

The power required to emit electron current from the cathode is

$$
P_{\text {cathode }}=I_{\text {anode }} V_{\text {gate }} \cdot
$$

The power dissipated in the tether, given by

$$
P_{\text {tether }}=I_{\text {tether }}^{2} R \text {, }
$$

is small relative to other losses because the current and resistance are low.

To solve for maximum available current, we assume that some fraction $\eta_{\text {anode-fraction }}$ of the overall generated power is reserved for current collection. The maximum current and anode potential can be calculated from Eq. (21) and Eq. (15). We then find the FEA base-gate potential $V_{\text {gate }}$ required to emit the collected current and calculate the power needed to operate the cathode.

The electromotive force is a loss mechanism for boosting in low inclination prograde orbits, but we disregard it in Eq. (19) because it is extremely small for the tether lengths considered. Current and power are directly related, so given the shape of the current-tether length curve in Fig. 8, we expect a similar shape for the power-tether length curve in Fig. 9. A short EDT requires a large current to overcome the drag force on the satellite. The rigidity of a beam decreases with length, so a very long EDT must have a large radius to prevent bowing. As a result, the drag due to the tether dominates over the drag due to the satellite, driving up the required current. The current is minimized when these two effects are balanced. Table 4 summarizes the power calculations.

Figure 9 shows the available power for thrust and the required thrust power. The available power is the estimated power available for EDT propulsion after DC-DC converter loss and loss to other loads. The required thrust power is the power needed to overcome the atmospheric drag force. If the required thrust power is higher than the available power, the EDT cannot overcome the drag force. However, if the converse is true and the femtosatellite has more power available than is required for thrust, the EDT can boost. 
Table 4. Power constraints for current collection.

\begin{tabular}{|l|c|c|c|}
\hline \multicolumn{1}{|c|}{ Parameter } & $\begin{array}{c}\mathbf{1 0 - m g} \\
\text { satellite }\end{array}$ & $\begin{array}{c}\mathbf{1 - g} \\
\text { satellite }\end{array}$ & $\begin{array}{c}\mathbf{1 0 0 - g} \\
\text { satellite }\end{array}$ \\
\hline Solar radiation flux $\left(\mathrm{W} \cdot \mathrm{m}^{-2}\right)$ & 1367 & 1367 & 1367 \\
\hline Earth albedo $(\%)$ & 30 & 30 & 30 \\
\hline Solar panel area exposed to the sun $\left(\mathrm{cm}^{2}\right)$ & 2 & 6 & 90 \\
\hline Total generated power $(\mathrm{mW})$ & 17 & 53 & 800 \\
\hline $\begin{array}{l}\text { Power available for propulsion after 25\% is lost } \\
\text { to DC-DC conversion and other loads }(\mathrm{mW})\end{array}$ & 13 & 40 & 600 \\
\hline Anode power $(\mathrm{mW})$ & 3.7 & 21 & 540 \\
\hline Equivalent anode sphere radius $(\mathrm{cm})$ & 0.7 & 0.86 & 1 \\
\hline Anode voltage $(\mathrm{V})$ & 17 & 52 & 430 \\
\hline Anode current $(\mu \mathrm{A})$ & 214 & 403 & 1250 \\
\hline Cathode power $(\mathrm{mW})$ & 8.5 & 16.7 & 56.6 \\
\hline Cathode base-gate voltage $(\mathrm{V})$ & 39.6 & 41.4 & 45.12 \\
\hline Power dissipated by tether $(\mathrm{mW})$ & 0.01 & 0.04 & 0.5 \\
\hline Total power consumed $(\mathrm{mW})$ & 12.2 & 37.7 & 596 \\
\hline
\end{tabular}

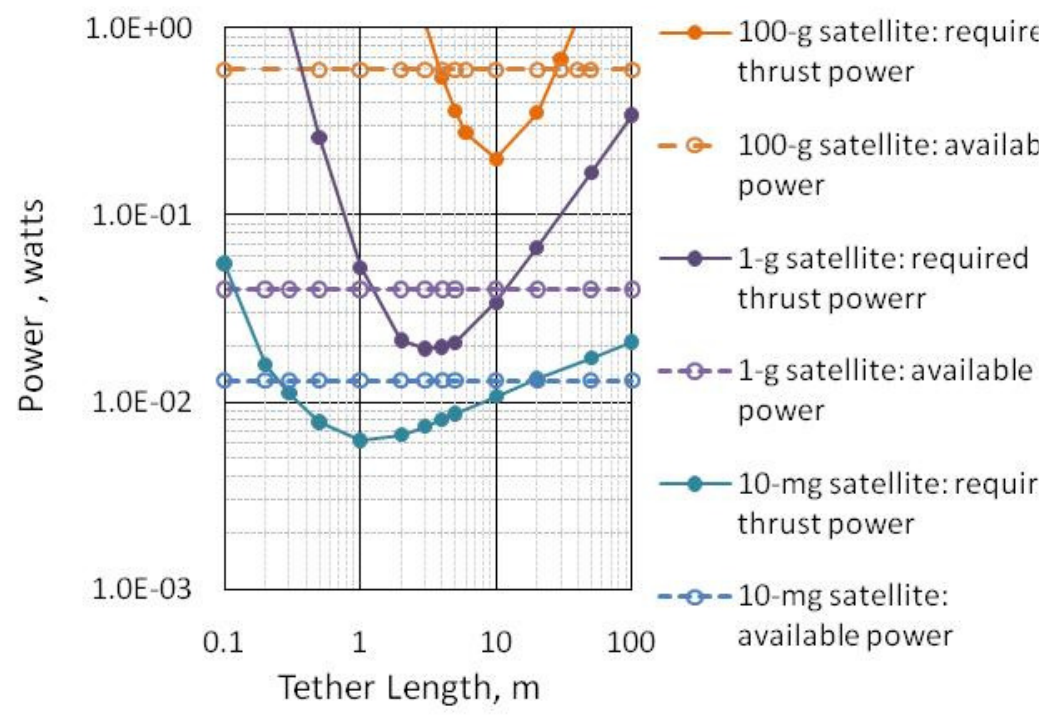

Figure 9. Electrical power required for drag make-up thrust and available electrical power. 


\section{Thrust Power as a Measure of Efficiency}

The instantaneous power expended generating EDT thrust is

$$
P_{\text {thrust }}=\mathbf{F}_{\text {Lorentz }} \bullet \mathbf{v}
$$

or, expanding the expression for the Lorentz force, is

$$
P_{\text {thrust }}=\left(I_{\text {tether }} \mathbf{L} \times \mathbf{B}\right) \bullet \mathbf{v} .
$$

We can also express the magnitude of the EDT thrust power in terms of the electromotive force as

$$
\left|P_{\text {thrust }}\right|=V_{\text {emf }} I_{\text {tether }} \cdot
$$

For an EDT operating in deboost mode in LEO, the electromotive force drives current in the tether, which converts orbital energy into electrical energy. In boost mode, however, the EDT system uses external energy, i.e., the solar energy converted to electrical energy and current flow, to increase the orbital energy of the satellite over time. For either deboost or boost mode, the overall EDT power losses include power used to collect and emit current in the ionosphere and power dissipated in ohmic loss in the tether. We can calculate the EDT thrust efficiency by taking the ratio of the thrust power and the total dissipated power, given by

$$
\text { Efficiency }=\frac{P_{\text {thrust }}}{P_{\text {total }}} \text {. }
$$

Figure 10 shows the thrust power efficiency, assuming the EDT system uses the total available power given in Table 4 for maximum thrust. The thrust efficiency increases with the tether length because the electromotive force increases with length. For tether lengths below 100 meters, a majority of the available power is dissipated in collecting current from the ionosphere and only a small percentage increases the energy of the orbit over time. The EDT system described here might be considered inefficient. However, the solar power available easily provides what is needed and the EDT is propellantless, so we believe the EDT still provides a potentially viable approach for propulsion.

The low efficiency reported here is in contrast to larger, more traditional EDT systems, which utilize much longer tethers and larger end-bodies. Larger end-bodies have more current collection area and can also make use of hollow cathodes for more efficient current collection, so a smaller fraction of the total power is dissipated to collect the required current. Longer tethers generate a higher electromotive force. By utilizing longer tethers and larger endbodies, the larger EDT systems are generally more efficient.

We may also need to revise our anode collection model. The collection theory does not calculate the exact current collected by the anode, so we may need to analyze and experimentally verify our assumptions to see if the current collection estimates here are too conservative. An anode that requires less voltage to collect the same current would be more efficient and could consequently raise the efficiency of the propulsion system.

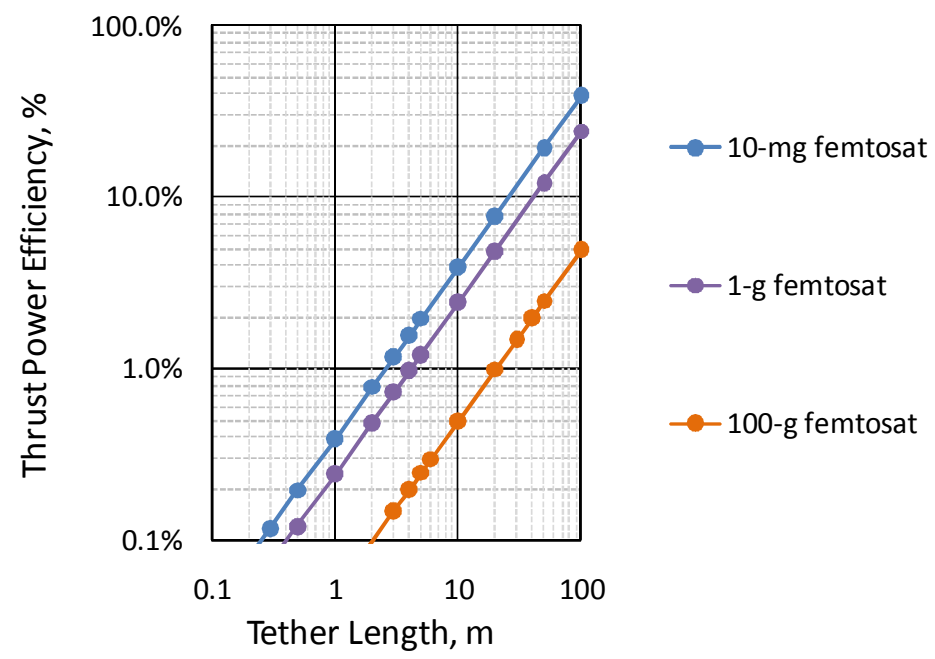

Figure 10. The percentage of available power used for thrust, assuming the total power available is used to achieve maximum thrust. 


\section{J. Estimating Forces on the Tether and the Femtosatellite}

The drag force, the solar radiation pressure force, the gravity gradient force, and the Lorentz force are the dominant forces acting on the femtosatellites and tether system in LEO. The drag force is given by Eq. (3), the gravity gradient force is given by Eq. (7), and the Lorentz force is given by Eq. (2). To estimate the solar radiation pressure force, we calculate the maximum force on each end-body a ${ }^{22}$

$$
F_{\text {plate }}=\frac{A \Phi_{\text {direct }}}{c}\left[\left(1-\Gamma_{\text {spec }}\right)+2\left(\frac{\Gamma_{\text {diffuse }}}{3}+\Gamma_{\text {spec }}\right)\right]
$$

and the maximum force on the tether as ${ }^{22}$

$$
F_{\text {cylinder }}=\frac{2 \pi r L \Phi_{\text {direct }}}{3 c}\left[\frac{1+\Gamma_{\text {spec }}}{\pi}+\frac{\Gamma_{\text {diffuse }}}{2}\right]
$$

and sum the quantities, assuming they simply add. Equations (28) and (29) are both maximum forces where the solar radiation is directly incident on the surface, i.e., there is no tilt angle between the surface normal and the incident solar radiation. The total solar radiation pressure force, shown in Figs. 11-13, is the sum of Eq. (28) and Eq. (29). We do not calculate the torque due to the solar radiation pressure force.

Table 5. Representative solar panel and Kapton optical properties.

\begin{tabular}{|c|c|c|}
\hline Parameter & Kapton H Film & Solar Panel $^{22}$ \\
\hline$\Gamma_{\text {spec }}$ & 0.104 & 0.042 \\
\hline$\Gamma_{\text {diffuse }}$ & 0.013 & 0.168 \\
\hline
\end{tabular}

Figures 11-13 show the forces that act on the tether-femtosatellite system. We expect the atmospheric drag force to be dominant for extremely small spacecrafts in LEO. ${ }^{24}$ EDT thrust exceeds the drag force for a range of tether lengths. To increase the boosting capability, we choose a tether length where there is the greatest difference between the drag force and the EDT thrust. This length also corresponds to the length that minimizes required current (Fig. 8) and power (Fig. 9).

Figures 11-13 also reveal that the margin between EDT thrust and drag is small for each of the satellites. The margin can be widened by increasing the EDT current and utilizing a thinner tether. A thinner tether would lower the drag force at the expense of tether rigidity. We could increase the current by revisiting our assumptions and determining if the current collection model and available power estimate are too conservative.

It should also be noted that the gravity-gradient force is very small relative to other forces for the 10-mg satellite and the 1-g satellite. The gravitygradient force generates tension along the tether and the torque aligns the tether along the local vertical. In this trade study, we assumed that the tether is aligned along the local vertical. For low inclination orbits, this alignment results in a maximum thrust given by Eq. (2). If we cannot assume vertical EDT orientation, the thrust should be calculated by Eq. (1). If the gravity gradient force is weak relative to the drag and solar pressure

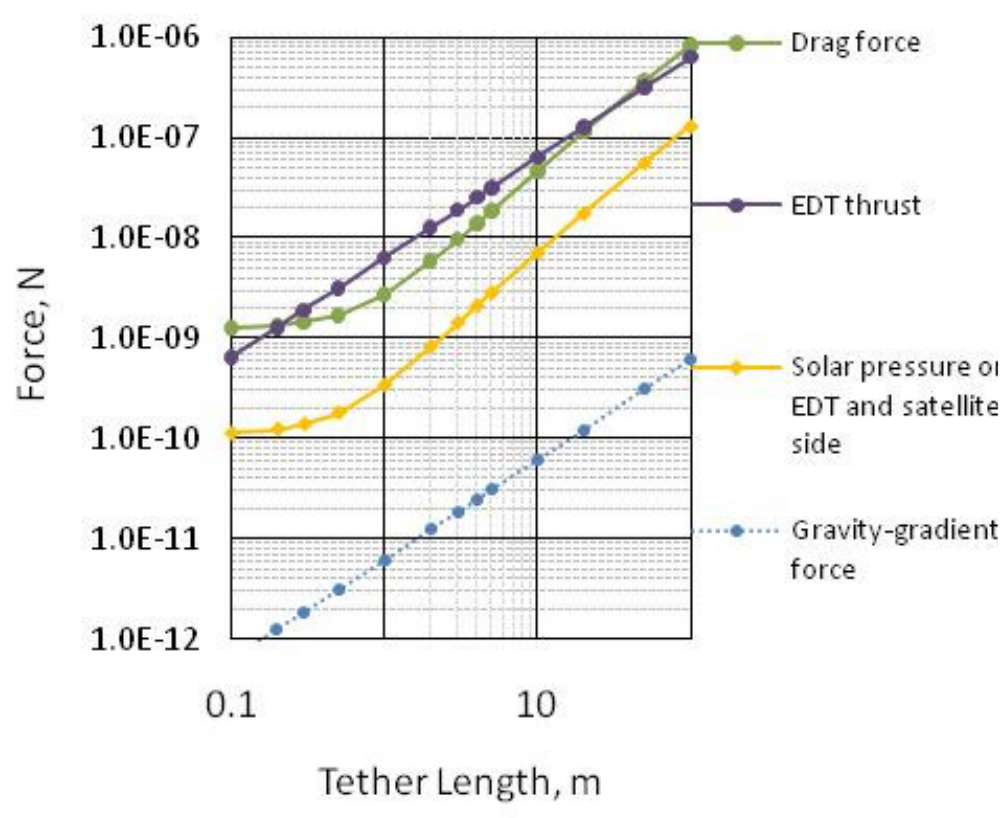

Figure 11. Forces on the 10-mg satellite and EDT. 
forces, we cannot assume any orientation. The magnitude and direction of the resulting EDT force can vary widely so drag make-up is not guaranteed, even with sufficient tether current.

For the 100-g satellite using a 10-m EDT, the gravity-gradient force is about twice the drag force. We assume that the spacecraft is aligned along the local vertical for the 10-m EDT because the gravity-gradient force is dominant. We need to analyze the spacecraft dynamics in more detail to accurately estimate the orientation.

The gravity-gradient force is small for the $1-\mathrm{g}$ satellite and negligible for the $10-\mathrm{mg}$ satellite, so we cannot assume that the system is aligned along the local vertical. In order to use EDT propulsion for these satellites, we either need to implement an alternative method for ensuring vertical EDT alignment or we can utilize tethers oriented along multiple spacecraft axes, as proposed in Ref. 25. By orienting tethers along different axes, we may be able to provide EDT thrust without gravity-gradient stabilization. With multiple tethers on different axes, the satellite can boost regardless of its orientation.

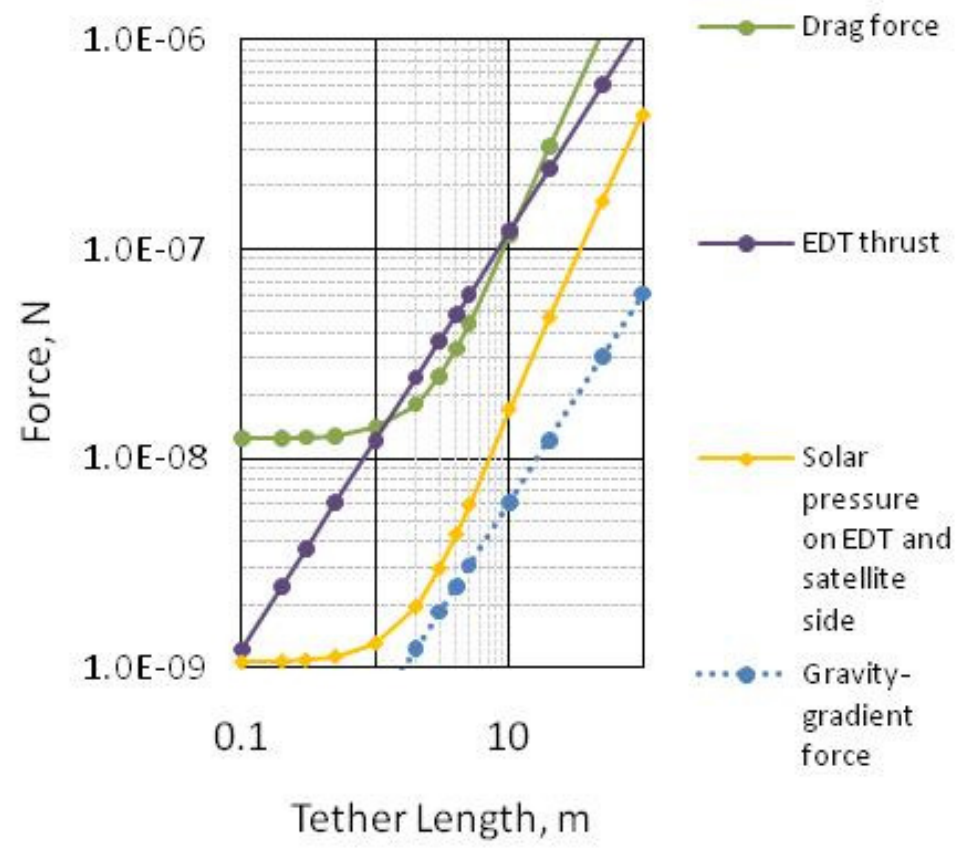

Figure 12. Forces on the 1-g satellite and EDT.

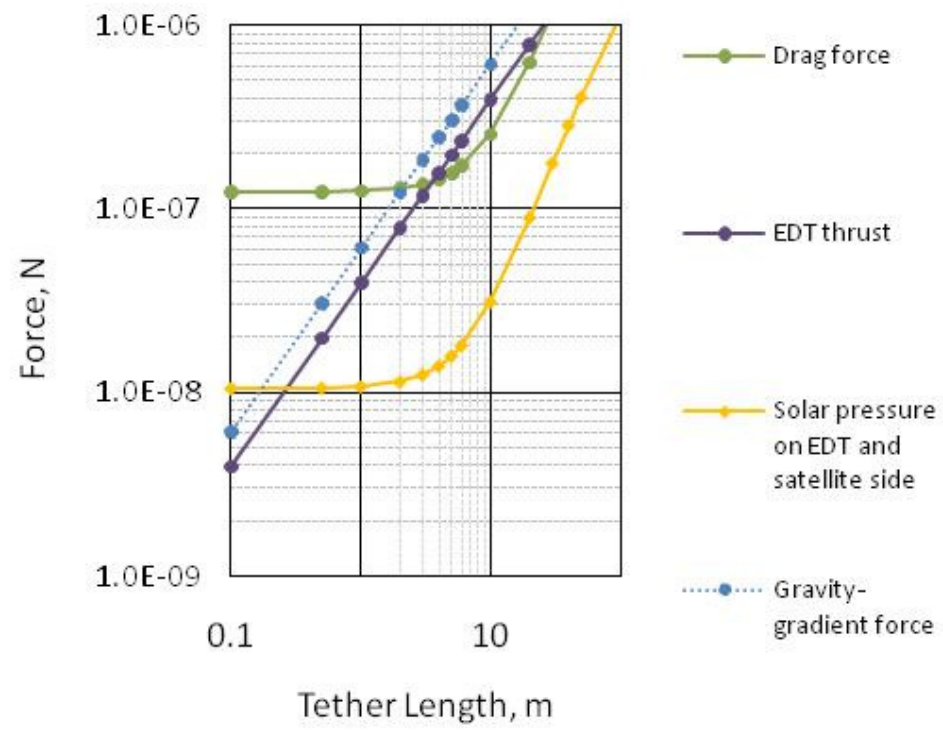

Figure 13. Forces on 100-g satellite and EDT.

American Institute of Aeronautics and Astronautics 


\section{K. Attitude Stability Concerns and Zeroth Order Estimates}

It is important to investigate the attitude stability of the EDT system because the EDT orientation affects thrust. In LEO in low inclination orbits, in-plane thrust requires the tether to be aligned near or along the local vertical. We need to understand if the gravity gradient torque will maintain stable orientation along the local vertical for all three femtosatellite and tether systems.

We first determine if the orientation of the EDT system along the local vertical is stable. We begin by positioning the EDT system according to Fig. 14 and assuming that the $x$ axis is aligned with the velocity vector, the $y$ axis is aligned along the orbit normal, and the $z$ axis is aligned with the nadir vector. In this coordinate system, a rotation about the $x$ axis is roll, a rotation about the $y$ axis is pitch, and a rotation about the $z$ axis is yaw. We model the EDT as a long, thin rod oriented along the $z$ axis and the end-bodies as relatively smaller cuboids with their largest dimensions in the $x y$ plane. We also know from the trade study that $L$ is much greater than both $w$ and $h$. Due to the proportions of the EDT system, the mass moment of inertia around the $z$ axis, or $I_{z}$, is much smaller than the mass moments of inertial around the $x$ or $y$ axis, or $I_{x}$ and $I_{y}$. The current design is also symmetric in the $x z$ and $y z$ planes, so $I_{x}=I_{y}$.

We can estimate attitude stability by comparing the relative sizes of the mass moments of inertia. The necessary and sufficient condition for pitch stability is $I_{x}>$ $I_{z},{ }^{26}$ so we can expect the EDT system to have stability in rotations around the $y$ axis. However, for roll and yaw stability, $I_{y}>I_{x}>I_{z},{ }^{26}$ so the EDT system does not have roll or yaw stability. We will need to study the mass moments of inertia and the conditions for attitude stability much more rigorously before we can draw more meaningful conclusions about the attitude dynamics of the femtosatellite and tether system.

We will also need to investigate the combined effects of gravity-gradient torque and torque due to atmospheric drag. Aerodynamic torque tends to orient the spacecraft so the velocity vector, $\mathbf{v}$, lies along the same line as the vector pointing from the center of mass to the center of pressure, $\mathbf{S}_{\mathrm{cp}}$. If the center of mass and

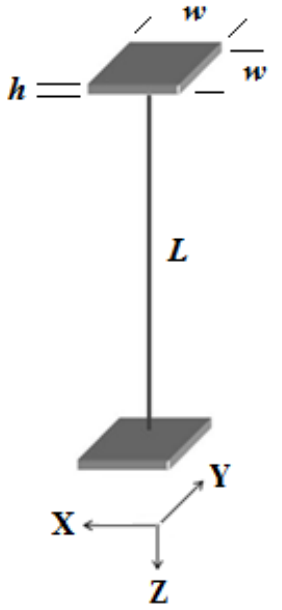

Figure 14. Femtosatellite Illustration with Axes. center of pressure are vertically displaced in a tether system, the aerodynamic drag torque will rotate the tether system away from the local vertical until $\mathbf{v} \| \mathbf{S}_{\mathrm{cp}}$, where the drag torque equals zero. The gravity-gradient torque, however, counteracts this rotation and tends to restore the tether to the local vertical. It is necessary to study the relative strength of the drag and gravity gradient torques in order to understand the resulting tether attitude.

\section{Preliminary experimental considerations}

It is essential to understand the capability of the anode to collect electron current because the current collected by the anode is directly proportional to EDT thrust. There is presently no theory that predicts the exact current collection to a biased cuboid in a flowing, weakly magnetized plasma. It is possible that the current collection estimate used in this trade study is too conservative and that we are underestimating EDT efficiency and underpredicting thrust capability. A laboratory experiment would provide a more accurate estimate of current collection by the anode and, as a result, would allow us to better estimate EDT performance in LEO. 


\section{Conclusion}

A short, semi-rigid EDT scales to the small size needed, is propellantless, keeps the overall ChipSat mass low, and provides enough thrust to overcome drag in LEO. For low inclination orbits, the EDT's ability to provide thrust requires the tether to be oriented along or near the local vertical. The gravity-gradient force is large relative to other forces for the 100-g femtosatellite. However, we are not prepared to assume that the gravity gradient torque will align the tether. Further analysis on attitude stability is continuing.

The gravity-gradient force for the $10-\mathrm{mg}$ and $1-\mathrm{g}$ satellites is small relative to the drag and solar pressure forces, so we cannot conclude that the EDT thrust capability is possible for a single EDT. An array of short tethers, however, oriented along different axes may be a feasible solution to EDT thrust for the small femtosatellites in the absence of gravity gradient stability. We will also continue to investigate anode current collection. Table 6 summarizes results from the trade study.

Table 6. Summary.

\begin{tabular}{|c|c|c|c|}
\hline Parameter & 10-mg Satellite & 1-g Satellite & 100-g Satellite \\
\hline Satellite dimensions & $1 \mathrm{~mm} \times 1 \mathrm{~cm} \times 1 \mathrm{~cm}$ & $1 \mathrm{~cm} \times 1 \mathrm{~cm} \times 1 \mathrm{~cm}$ & $2 \mathrm{~cm} \times 5 \mathrm{~cm} \times 5 \mathrm{~cm}$ \\
\hline Ram drag cross sectional area & $0.1 \mathrm{~cm}^{2}$ & $1 \mathrm{~cm}^{2}$ & $10 \mathrm{~cm}^{2}$ \\
\hline EDT length & $1 \mathrm{~m}$ & $3 \mathrm{~m}$ & $10 \mathrm{~m}$ \\
\hline Tether diameter & $24 \mu \mathrm{m}$ & $66 \mu \mathrm{m}$ & $212 \mu \mathrm{m}$ \\
\hline Tether mass & $3 \mathrm{mg}$ & $80 \mathrm{mg}$ & $3 \mathrm{~g}$ \\
\hline Available power (estimated) & $13 \mathrm{~mW}$ & $40 \mathrm{~mW}$ & $600 \mathrm{~mW}$ \\
\hline Current & $214 \mu \mathrm{A}$ & $403 \mu \mathrm{A}$ & $1250 \mu \mathrm{A}$ \\
\hline Thrust power efficiency & $0.4 \%$ & $0.7 \%$ & $0.5 \%$ \\
\hline $\begin{array}{c}\text { Is gravity gradient force } \\
\text { significant? }\end{array}$ & $\mathrm{no}$ & $\mathrm{no}$ & yes \\
\hline
\end{tabular}




\section{Acknowledgments}

The authors gratefully acknowledge support from AFOSR grant FA9550-09-1-0646. I. C. Bell would also like thank J. Rager his enormous contribution to this study.

\section{References}

${ }^{1}$ Atchison, J. and Peck, M., “A Passive, Sun-Pointing, Millimeter-Scale Solar Sail,” Acta Astronautica, Vol. 67, No. 1, pp. $108-121,2010$.

${ }^{2}$ Barnhart, D., Vladimirova, T., and Sweeting, M., "Very-small-satellite Design for Distributed Space Missions," Journal of Spacecraft and Rockets, Vol. 44, No. 6, 2007, pp. 1294-1306.

${ }^{3}$ Barnhart, D., Vladimirova, T., and Sweeting, M., "Satellite-on- a-chip Development for Future Distributed Space Missions," Proceedings of the CANEUS Conference on Micro-Nano Technologies for Aerospace Applications, Toulouse, France, 2006.

${ }^{4}$ Barnhart, D.J., "Very Small Satellite Design for Space Sensor Networks," Presentation, Brown University ChipSat Workshop, Providence, RI, Feb. 18, 2010.

${ }^{5}$ Peck, M. A., “A Vision for Milligram-scale Spacecraft,” Presentation, Brown University ChipSat Workshop, Providence, RI, Feb. 18, 2010.

${ }^{6}$ Tang, W. C., "Overview of MEMS Programs at DARPA and Applications in Space," Presentation, First Canadian Workshop on MEMS Technology for Aerospace Applications, National Research Council, Canada, April 12, 2001.

${ }^{7}$ Bell, I. C., "Electrodynamic Tethers for ChipSat and Nanospacecrafts," Poster, Spacecraft Charging and Technology Conference, Albuquerque, NM, Sept. 23, 2010.

${ }^{8}$ Fuhrhop, K.R.P., "Theory and Experimental Evaluation of Electrodynamic Tether Systems and Related Technologies." Ph.D. Thesis, The University of Michigan, pp. 1-53, 2007.

${ }^{9}$ Bell, I.C. et al., "The Potential of Miniature Electrodynamic Tethers to Enhance Capabilities of Femtosatellites" $32{ }^{\text {nd }}$ International Electric Propulsion Conference, Wiesbaden, Germany September 2011.

${ }^{10}$ Larson, W. and Wertz, J. (eds.), Space Mission Analysis and Design, Microcosm, Inc., 1992.

${ }^{11}$ Willson, R. C., and A. V. Mordvinov, "Secular Total Solar Irradiance Trend During Solar Cycles 21-23," Geophys. Res. Lett., Vol. 30, No. 5, p. 1199, 2003.

${ }^{12}$ Hinkley, D., "Picosatellites at The Aerospace Corporation," Chapter 20 in Small Satellites: Past, Present, and Future, edited by H. Helvajian and S. W. Janson (The Aerospace Press and the AIAA, El Segundo, CA, 2009).

${ }^{13}$ Cosmo, M.L. and Lorenzini, E.C., "Tethers in Space Handbook," 2nd Ed., prepared for NASA/MSFC by Smithsonian Astrophysical Observatory, Cambridge, MA, 1989.

${ }^{14}$ Davis, J.R., Nickel, Cobalt, and Their Alloys. ASM International, Materials Park, OH, pp. 26-27, 2000.

${ }^{15}$ Dias, J.F., Bulla, A., and Yoneama, M.L., "Charging Effects in Thick Insulating Samples," Nucl. Instr. and Meth., Vol. B 189, 2002.

${ }^{16}$ Hibbeler, R.C., Mechanics of Material, 6th Ed., 2000, Prentice Hall International, Inc, Chaps. 6 and 12.

${ }^{17}$ Rubinstein, J. and Laframboise, J. G., "Theory of a Spherical Probe in a Collisionless Magnetoplasma” Phys. Fluids, Vol. 25, p. 1174, July 1982.

${ }^{18}$ Mott-Smith, H.M. and Langmuir, I. “The Theory of Collectors in Gaseous Discharges,” Phys. Rev., Vol. 28, pp. 727-763, 1926.

${ }^{19}$ Parker, L W., and Murphy, B.L., "Potential Buildup on an Electron Emitting Ionospheric Satellite," J. Geophys. Res., Vol. 72, p. 1631, 1967.

${ }^{20}$ Whaley, D., et al., "100 W Operation of a Cold Cathode TWT," IEEE Trans. Electron Devices, Vol. 56, No. 5, pp. 896905, May 2009.

${ }^{21}$ Warneke, B.A., et al., "An Autonomous $16 \mathrm{~mm}^{3}$ Solar-powered Node for Distributed Wireless Sensor Networks," IEEE Sensors 2002 Proceedings, Vol. 2, p. 1510-1515, 2002.

${ }^{22}$ Kubo-oka, T. and Sengoku, A., "Solar Radiation Pressure Model for the Relay Satellite of SELENE," Earth Planets Space, Vol. 51, pp. 979-986, September 1999.

${ }^{23}$ Waters, D.L., Groh, K., Banks, B., and Cameron, K., "Changes in Optical and Thermal Properties of the MISSE 2 Peace Polymers and Spacecraft Silicones" International Symposium on Materials in a Space Environment, September 18, 2009.

${ }^{24}$ Atchison, J. and Peck, M., "Length Scaling in Spacecraft Dynamics," Journal of Guidance, Control, and Dynamics, Vol. 34, No. 1, pp. 231-246, 2011.

${ }^{25}$ Voronka, N., Hoyt, R.P., Gilchrist, B. E., and Fuhrhop, K., “An Architecture of Modular Spacecraft with Integrated Structural Electrodynamic Propulsion (ISEP)," NIAC 7 the Annual Meeting, Tucson, AZ, 18 October 2006.

${ }^{26}$ Hughes, P. C., Spacecraft attitude dynamics, Dover ed., Mineola, New York, 2004, Chaps. 7, 14. 